\title{
Neural Network Based Enveloping Model of Agricultural Tyre
}

\author{
B. STоJIĆ \\ University of Novi Sad, Faculty of Technical Sciences, bstojic@unc.ac.rs
}

\begin{abstract}
The vibration properties of agricultural tractor's tyres significantly influence its response in terms of the exposure of the human operator to mechanical vibrations, which is still one of the key problems in the design and exploitation of tractors. The behaviour of the tyre in this sense is significantly influenced by the mechanism of geometric filtering of the short-wavelength unevenness of the unprepared ground mostly encountered by tractor during in-field operations. Herein, this aspect of tyre behaviour was studied with tyre rolling quasistatically over singular obstacle. Based on experimental results, neural network based model of tyre enveloping behaviour was developed. Main model properties are high computational efficiency, simplicity regarding number of input quantities and absence of the need for explicit parametrization etc. Main shortcomings of modelling approach used are high developing effort, labour-intensive experimental preparations and lack of flexibility regarding tyre design and operating parameters.
\end{abstract}

\section{Introduction}

The vibration properties of agricultural tractor's tyres significantly influence its response in terms of the exposure of the human operator to mechanical vibrations, which is still one of the key problems in the design and exploitation of tractors. The need to solve this problem plays a significant role in the process of developing and optimizing the tractor's design parameters. The behaviour of the tyre in this sense is significantly influenced by the mechanism of geometric filtering of the short-wavelength unevenness of the unprepared ground mostly encountered by tractor during in-field operations. Herein, this aspect of tyre behaviour was studied by using singular road obstacle of rectangular shape. Experiments were carried out with tyre rolling quasistatically in order to eliminate all other kinds of influences and to isolate geometric filtering from other mechanisms manifested by the tyre.

Tyre enveloping behaviour is result of local stress-strain state of its structure, which is governed by complex physical mechanisms. Tyre response in this view is defined by phenomena such as anisotropic behaviour of the composite structure, viscoelasticity of rubber compound, large 3D strain with appropriate stress state leading to pronounced non-linear behaviour, etc. Because of this, dealing with such behaviour by using analytical approach can be rather cumbersome. It can therefore be of interest to study this aspect of tyre behaviour by means of physical experiments instead of using analytical approach. Experimental results can then be used to derive appropriate empirical model. It is in certain cases advantageous to use such approach when analysis is focused on vehicle dynamics rather than on the tyre itself. Under such circumstances, there is often a need for tyre model with 
relatively high computational speed, especially in cases when the purpose of the simulation is development or governing of vehicle dynamics control systems [7], [9]. This requirement usually cannot be achieved by using analytical modelling approach for the tyre, because the physical model has to be of a similar level of complexity as the natural phenomena being described by the model. Empirical approach, overlooking physical nature of the system in regard, can hence lead to significantly faster model.

Aim of this work is to develop enveloping model of the tractor tyre by using of artificial neural network for empirical modelling, in order to overcome constraints and difficulties of physical modelling approach. Thereafter, model properties in terms of accuracy, computing speed, developing effort, flexibility to change of input data etc. will be analysed and discussed.

\section{Experimental setup}

\subsection{Test facility}

Experimental measurements required to obtain empirical data were conducted by using test facility whose simplified scheme is depicted in the Figure 1. Basically, facility is based on the rail-guided cart in which tested wheel is mounted so that it can roll along the testing ground. Wheel thereby has freedom to move in vertical direction when negotiating road unevenness or obstacles, so that ground force remains constant if the quasi-static regime of motion is applied. Measuring vertical rim displacement vs. longitudinal wheel position, effective road profile curves are obtained. Test facility is described in more details in previous papers, e.g. [11].

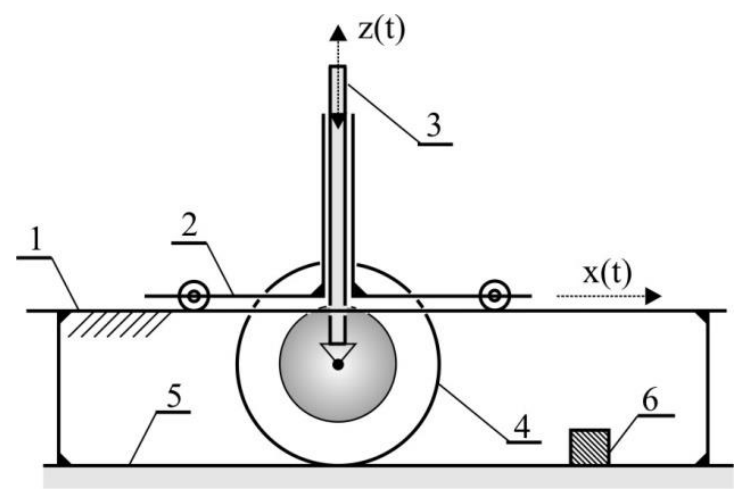

Figure 1 Simplified schematic view of the test facility: 1-cart guiding rail, 2-wheel guiding cart, 3-vertical wheel guide, 4-tested wheel, 5-level ground, 6-road obstacle; $x(t), z(t)$-longitudinal and vertical travel of the wheel rim

\subsection{Testing conditions}

Experimental investigations were carried out by quasi-static rolling of the tyre over the ground obstacle. By the term "quasi-static" it is meant that the longitudinal velocity of the wheel was small enough that all dynamic effects (i.e. all manifestations of inertia and damping) were of such order of magnitude that their contribution to measuring results could be neglected. 
For the ground obstacle, rectangular shape of the cross section has been selected. Obstacles of different dimensions in view of height and length were used, height varying between 50 and $150 \mathrm{~mm}$ and length between 100 and $300 \mathrm{~mm}$. As it was shown in previous investigations [12] that tyre contact length can be used as single operating parameter replacing different combinations of pressure and load, three levels of contact length were used for experimental measurements.

Dimensions of the tyre used for investigations were 12.4R28.

\section{Experimental results}

Typical qualitative shapes of the output curves for different operating conditions are shown in the Figure 2. Curves represent paths of the wheel centre when tyre rolls quasistatically over rectangular road obstacle. This wheel motion introduces effective vibration excitation to the tractor. Case $(a)$ corresponds to large contact length i.e. large tyre load or low pressure. From case $(a)$ to case $(c)$ contact length decreases, which corresponds to load decrease and/or pressure increase.

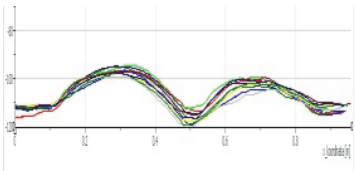

a)

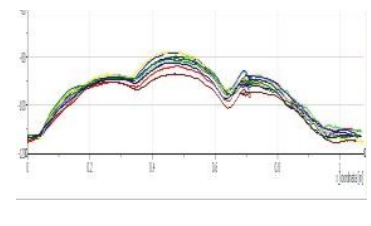

b)

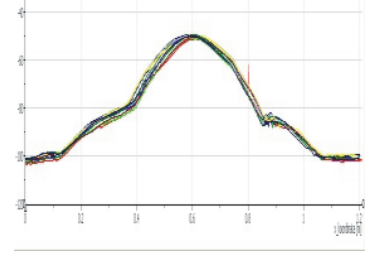

c)

Figure 2 Output curves for different operating conditions - qualitative view: large (a), moderate (b) and small (c) tyre contact length as working parameter

Output curve is described in more details in the Figure 3. Case (a) depicts geometrical parameters and relationship between input (which is rectangular obstacle) and output curve (wheel travel path). In case $(b)$, direct comparison between curves is shown with real dimensions, as obtained for one pair of obstacle dimensions (300x100 $\mathrm{mm})$ and tyre contact length $(280 \mathrm{~mm})$. Symmetry of curves with respect to vertical axis allows using view of only first (leading) half of the curves, trailing half looking the same but mirrored.

a)
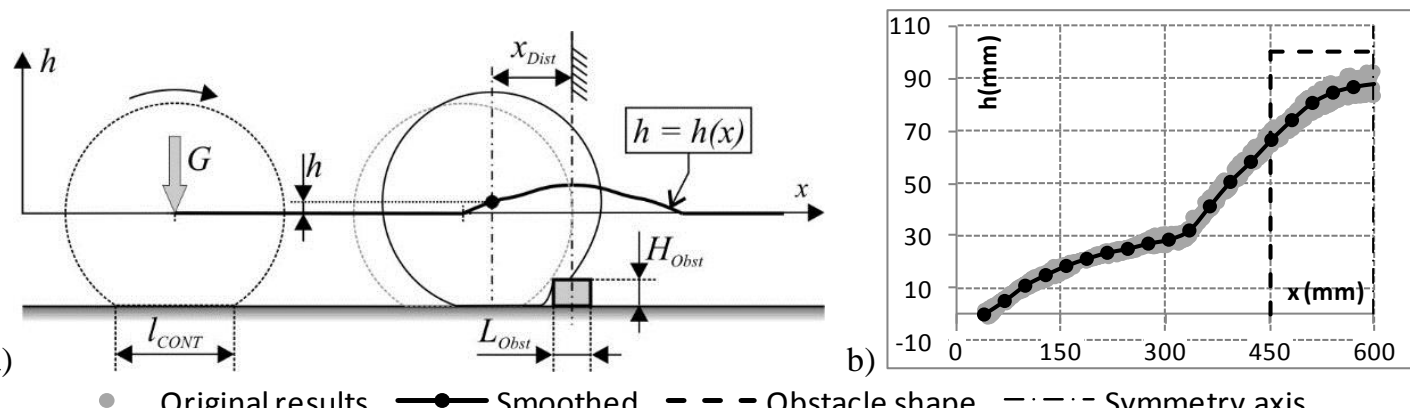

Figure 3 Example of the tyre enveloping curve for the singular ground obstacle of rectangular cross section: $h$ effective road profile height, $x$ - longitudinal wheel travel, $h=h(x)$ - enveloping curve, $G$ - vertical force exerted at the wheel, $l_{\text {ConT }}$ - tyre contact length on the level ground, $x_{\text {Dist }}$ - wheel position with respect to the obstacle, $L_{\text {Obst }}, H_{\text {obst }}$ obstacle dimensions; b) Graphical representations of typical test results (only first half of input and output curve shown, i.e. up to the vertical axis of symmetry) 


\section{Tyre enveloping model}

A number of models focused on, or capable of, describing tyre enveloping behaviour have been developed previously, mainly based on physical or semi-physical approaches. Here several examples are listed, such as structural modelling [5], adaptive footprint approach [1][4][13], basic functions and/or contact followers technique [2][10][14], FEM technique [6] etc. Empirical technique was used in frequency domain [15]. When developing tyre model able to take ground profile filtering mechanism into account, one can distinguish between physical and empirical approach. First one, taking into account tyre structure and nature, is more flexible and comprehensive, but on the account of complexity and slow execution. Empirical approach, on the other hand, does not have advantages of analytical methods, but can be far more computationally efficient which makes it especially appropriate for usage in vehicle dynamics simulations. Topic of this paper is development of an empirical model of tractor tyre enveloping behaviour based on the artificial neural network, by which the contact of the tyre with a surface with pronounced short-wavelength undulations is taken into account. Concept of model principle and application is illustrated in the Figure 4. According to this approach, overall tyre model is separated into parts of which one serves to describe contact of the tyre with uneven ground, while the other describes dynamic response of the viscoelastic structure of the tyre (Figure $4(a)$ ). Since behaviour and modelling of the later is out of the scope of this paper, most simple approach of using Voigt-Kelvin model (elastic and viscous element connected in-parallel) is used in the figure for the purpose of illustration.

a)

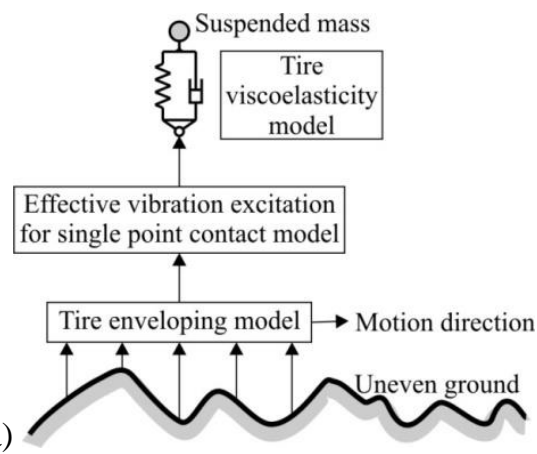

b) $L_{2}$

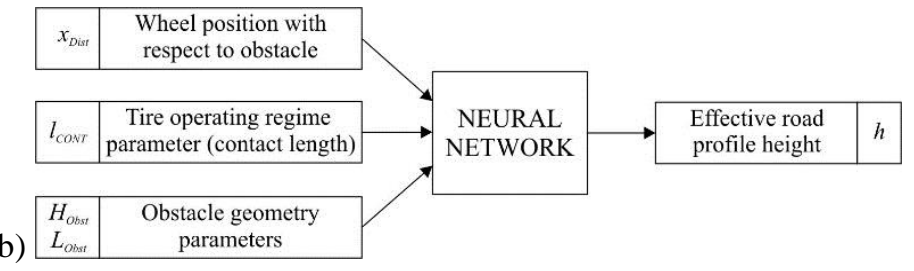

Figure 4 a) Schematic view of separation of the tyre model into parts responsible for road profile filtering and for tyre viscoelasticity; b) Structure of NN based empirical model of tyre enveloping behaviour for rectangular shaped obstacle

Tyre enveloping model is therefore integral part of overall tyre model, hence consisting of this part itself and the part that describes response of the viscoelastic structure of the tyre. Two parts interact in way that the first one generates effective vibration excitation for the second one, which then describes tyre dynamics, giving at the same time feed-back information to the enveloping model. The main purpose of this feed-back signal is to define input data for enveloping model in view of tyre operating regime through some appropriate parameter, which may be e.g. ground force, or its equivalents such as radial deformation or contact length.

Principle of the neural-network based enveloping model is shown in the Figure $4(b)$. Input parameters of this model are tyre contact length (obtained by the other part of the tyre viscoelastic model), obstacle dimensions in terms of height and length, and current longitudinal position of the 
wheel with respect to the obstacle. As output, model gives current ordinate value of the wheel travel path.

\section{Neural network model development}

\subsection{Basic characteristics of the development process}

Since the task of the neural network is the realization of the empirical model, i.e. the unambiguous generation of the output result based on the selected set of input data, feed-forward backpropagation network with supervised learning process was considered as an optimal approach for the given modelling task [3][8]. The general structure of such network is shown in Figure 5, taking into account the structure of the input and output data according to the Figure 4: 4 input data (length and height of the obstacle, longitudinal position of the wheel, tyre contact length) and 1 output (the ordinate of the effective ground profile, i.e. vertical position of the wheel).

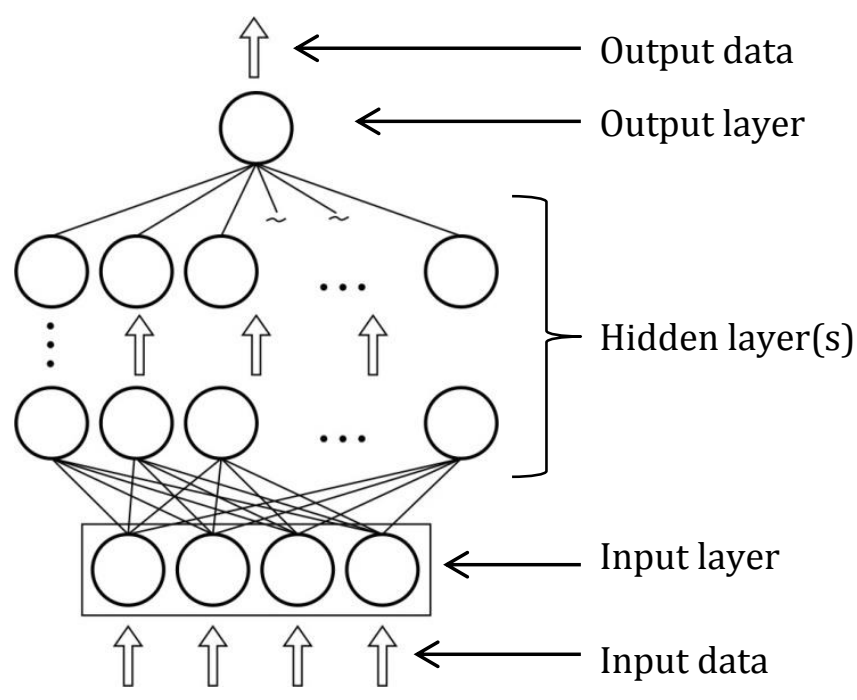

Figure 5 General structure of feed-forward backpropagation neural network

Since the numbers of neurons in the input and output layers are determined by the model structure (one neuron is the carrier of a single data), the problem of optimizing the topology of the network is reduced to determining the optimal number of hidden layers and the number of neurons therein, as well as the number and structure of synaptic connections.

The optimal choice of the topology of a neural network from the aspect of modelling the regarded system or phenomenon is a problem for whose solution there is no uniquely defined approach. Therefore, training needs to be carried out for different network configurations to determine experimentally which of them gives best results. The similar can be said for the selection of sets of empirical data communicated to the neural network within the learning process. Empirical data should be selected in such a way as to adequately represent the scope of the cases for which the model is to be applied, which is also a requirement for whose solution there is no uniquely defined approach.

During training of the neural network, the criterion for selecting the optimal number of training cycles represents the minimum error of the validation set, which is used to assess the ability of the network 
to generalize. This information, however, is by itself not sufficient to evaluate the validity of a neural network as an empirical model. For the final assessment it is necessary to quantitatively and qualitatively check the matching of the network output with the experimental results of those sets of data not used in the training process. It is especially important to take into account the occurrence of the "overtraining" of the neural network, that is, the phenomenon that, due to excessive adjustment of synaptic coefficients to data sets used in the training process, network completely loses the ability to generalize. In other words, this means that the model gives poor predictions for input data that were not included in the training process.

Only after such a check is conducted for a number of trained neural networks of different configurations, it is possible to determine which of them in an optimal way meets the given requirements. The process of comparing experimental data with predictions of an empirical model for data sets that are not used in the scope of the training process actually represents the validation of the model. From all of the above, it can be concluded that validation is an integral part of the model development process when creating a model based on an artificial neural network. Thus, these models differ from conventional empirical modelling techniques in which validation is carried out as a separate stage.

\subsection{Optimal neural network topology}

Based on previous considerations, neural network training process has been carried out with many different network topologies, whereby main changing parameters were number of hidden layers and number of neurons therein. Different ways of interconnections between layers and individual neurons have also been tried. In all cases, predictions of different neural networks were compared both quantitatively (in term of error RMS) and qualitatively (in term of agreement between key geometrical properties of predicted and measured output curve shape). Using these criteria, final choice of optimal neural network was made.

Characteristics of neural network adopted as optimal model structure were as following: type of network - feed-forward, back propagation; number of input neurons - 4 (wheel position, tyre contact length, obstacle height, obstacle length); number of hidden layers - 1; number of neurons in hidden layer - 8; number of output neurons - 1 (effective road profile height); connectivity - forward directed, full connectivity of adjacent layers; training algorithm - Standard Backpropagation algorithm; activation function - Logistic function, $f(x)=1 /\left(1+e^{-x}\right)$. Several examples of model prediction of effective road profile, compared to experiment result, are shown in the Figure 6. It can be seen that model gives generally good predictions, although in some cases there are some discrepancies that can be described as more of the qualitative nature. 

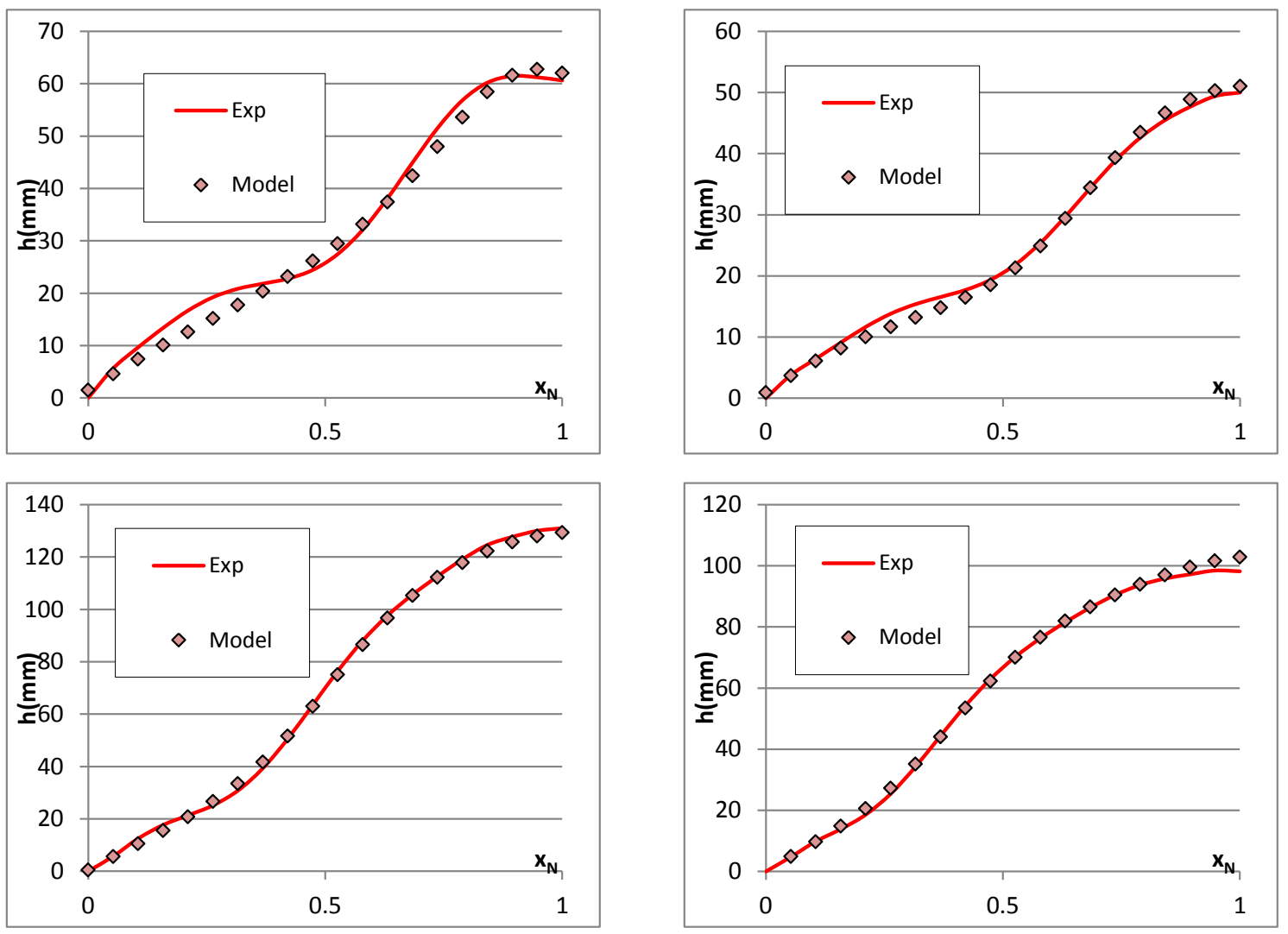

Figure 6 Example of model prediction of effective road profile $h$, compared to experiment results ( $x_{N}-$ normalized longitudinal position of the tyre with respect to the obstacle, value "1" meaning coinciding vertical axes of symmetry of both tyre and rectangular obstacle)

\subsection{Main model characteristics}

Neural network based model is characterized by high computational speed, whereby it fulfils this important requirement for use in vehicle dynamics simulations. Other main requirements in this view, accuracy and generalization ability are fulfilled in satisfactory level but through future work it should be checked out whether these can be further improved. As main characteristics of the model, the following can be named:

- High computational efficiency;

- Versatility, i.e. modelling approach can be used for other kind of tyres as well, since experimental investigations shown that tractor tyres behave similarly to other tyres in view of enveloping behaviour;

- Simplicity and few significant input quantities, contributing to high computational speed;

- Satisfactory accuracy and generalization ability;

- Model parameterization is carried out automatically, parameters (synaptic coefficients values) are hidden from user so there is no need for explicit parameterization procedure which makes model use more simple;

- Developing effort is high since there are no unambiguous criteria for optimal choice of neural network topology, training data set etc. so a lot of work has to be carried out based on the method of trial and error; 
- Intensive experimental work on the collecting and processing empirical data for neural network training is necessary;

- Lack of flexibility regarding tyre design changes, since no connection exists between physical properties of the tyre and model parameters.

\section{Conclusions}

In this paper development of agricultural tyre enveloping model for description of tyre contact with uneven ground, based on artificial neural network, was described. This approach was adopted mainly in order to overcome constraints and difficulties in physical modelling of the tyre behaviour. General properties of artificial neural networks are well suited for highly complex and non-linear behaviour exhibited by agricultural tyres. It was shown that model gives good prediction although in some cases accuracy of results was questionable, though perhaps more from the qualitative point of view.

Main model properties are high computational efficiency, simplicity regarding number of input quantities and absence of the need for explicit parametrization etc. Main shortcomings of modelling approach used are high developing effort, labour-intensive experimental preparations and lack of flexibility regarding tyre design and operating parameters.

In the scope of further work, this enveloping model should be integrated into comprehensive tyre model consisting of enveloping model and structure model, and procedure for application of such model in the scope of tractor vibration dynamics simulation developed. Further model development in order to enhance accuracy should be carried out. Adjusting model to be able to accept more general shapes of the ground profile geometry should also be considered.

\section{References}

[1] J. M. Badalamenti - G. R. Doyle (1988) Radial-Interradial Spring Tire Models. Journal of Vibration. Acoustics, Stress and Reliability in Design, 110 (1) pp. 70-75

[2] P. Bandel - C. Monguzzi (1988) Simulation model of the dynamic behaviour of a tire running over an obstacle. Tire Science and Technology. 16 (2) pp. 62-77

[3] A. Barber (1999) Accurate Models for Bushings and Dampers using the Empirical Dynamics Method. 14th European ADAMS Users' Conference, Berlin.

[4] K. M. Captain - D. N. Wormley - E. Grande (1974) The Development and Comparative Evaluation of Analytical Tire Models for Dynamic Vehicle Simulation. U. S. Army TACOM.

[5] S. Gong (1993) A Study of In-Plane Dynamics of Tires. Doctoral thesis, Delft University of Technology.

[6] M. A. Erşahin (2003) Finite Element Analysis of Cornering Characteristics of Rotating Tires. PhD Thesis, Middle-east Technical University, Ankara.

[7] S. Herkt (2008) Towards a Finite Element Tyre Model for Multibody Simulation. Dissertation, Universität Kaiserslautern.

[8] W. Kinnebrock (1992) Neuronale Netze - Grundlagen, Anwendungen, Beispile. R. Oldenbourg Verlag München Wien. 
[9] F. Oueslati (1995) An Analytical Investigation of Passive and Active Suspension Systems for Articulated Freight Vehicles. PhD thesis, Concordia University Montreal, Quebec.

[10] A. J. C. Schmeitz (2004) A Semi-Empirical Three-Dimensional Model of the Pneumatic Tyre Rolling over Arbitrarily Uneven Road Surfaces. Doctoral thesis, Delft University of Technology.

[11] B. Stojić - N. Poznanović - A. Poznić (2017) Research and Modeling of the Tractor Tire Enveloping Behavior. Journal of Vibration and Control. 23(2) pp. 290-304

[12] B. Stojić (2014) Istraživanje mogućnosti korišćenja kontaktne dužine traktorskog pneumatika kao jedinstvenog eksploatacionog parametra envelope kretanja ("Investigation of the possibility of using the contact length of a tractor tire as a unique working parameter of tyre enveloping behaviour"). Traktori i pogonske mašine. 19 (3) pp. 23-28

[13] P. Witzel - S. Böttinger (2011) Upgrading of the Hohenheim Tyre Model to a radial approach for the simulation of obstacle passages. VDI Berichte Nr. 2124, VDI-Verlag, Düsseldorf. pp. 431-437

[14] P. W. A. Zegelaar (1998) The Dynamic Response of Tyres to Brake Torque Variations and Road Unevennesses. PhD Thesis, Technical University of Delft, Netherlands, Delft, NL.

[15] А. А. Хачатуров (1976) Динамика системы дорога - шина - автомобиль - водитель ("Dynamics of the system road - tire - car - driver"). Машиностроение. 